NBSIR 84-3015

\title{
A PRELIMINARY INVESTIGATION INTO USING THE SUN AS A SOURCE FOR G/T MEASUREMENTS
}

National Bureau of Standards

U.S. Department of Commerce

Boulder, Colorado 80303

August 1984

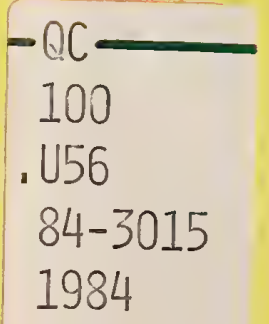





\section{A PRELIMINARY INVESTIGATION INTO USING THE SUN AS A SOURCE FOR G/T MEASUREMENTS}

William C. Daywitt

Electromagnetic Fields Division National Engineering Laboratory National Bureau of Standards U.S. Department of Commerce Boulder, Colorado 80303

August 1984

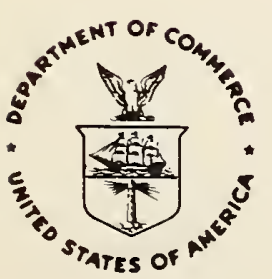

U.S. DEPARTMENT OF COMMERCE, Malcolm Baldrige, Secretary 



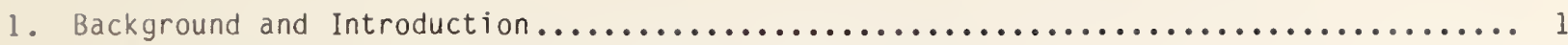

2. Determination of the Solar Flux Density Above $5 \mathrm{GHz} \ldots \ldots \ldots \ldots \ldots$

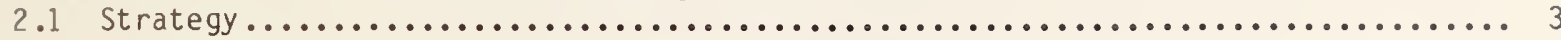

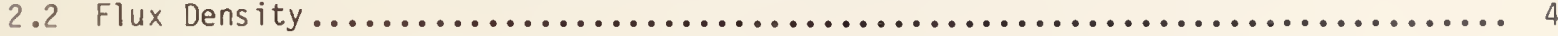

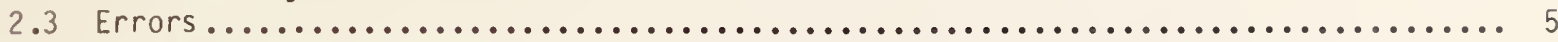

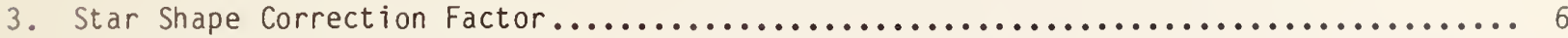

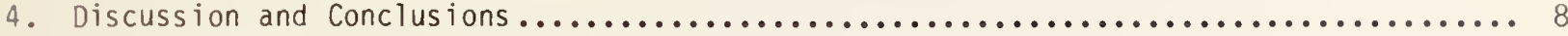

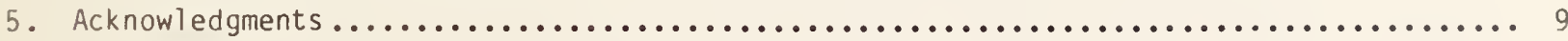

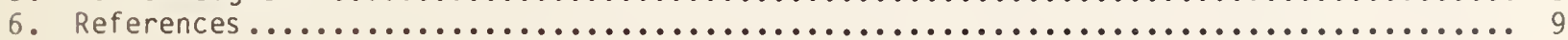



A Preliminary Investigation Into Using the Sun as a Source for G/T Measurements

\author{
William C. Daywitt \\ Electromagnetic Fields Division \\ National Bureau of Standards \\ Boulder, Colorado 80303
}

This report describes a preliminary investigation into determining the solar flux density, the atmospheric correction factor, and the star shape correction factor for use in $G / T$ measurements above $5 \mathrm{GHz}$. An estimate of errors is also included. Preliminary results show: an improved algorithm for determining diffusive and refractive attenuation; a viable technique for estimating the solar flux density from daily AFGL flux density measurements and a centimeter/millimeter wave spectrum function; and the possibility of reducing star shape correction factor errors by use of an effective solar rf diameter.

Key words: atmospheric correction factor; earth terminal measurement system; error analysis; G/T; solar flux density; star shape correction factor.

\title{
1. Background and Int roduction
}

The Electromagnetic Fields Division of the National Bureau of Standards has successfully made precision G/T (gain to system noise temperature) measurements [1] on low noise, large diameter $(11 \mathrm{~m}$ to $28 \mathrm{~m}$ ) antenna systems using the radio star Cassiopeia $\mathrm{A}$, and on medium size $(4 \mathrm{~m}$ to $6 \mathrm{~m})$ antenna systems using the moon. Although the moon is an attractive source to use with these smaller systems because of its predictable radio output [2], in many cases [3] the system noise is so much greater than the received lunar output that the measurement sensitivity is too low to be useful. Since the solar angular diameter is about that of the moon and its output is considerably greater, the sun provides an answer to the sensitivity problem. Unfortunately, its flux density and brightness distribution are variable, and even with this preliminary study a number of investigations are still needed to determine the correct G/T measurement error. This report describes an effort to determine the feasibility of using the daily Air Force Geophysics Laboratory (AFGL) flux density values [4] measured at the Air Force Sagamore Hill Observatory in Hamilton, Massachusetts. The results pertain to frequencies above $5 \mathrm{GHz}$ for reasons that will be made clear later.

The antenna system $G / T$ can be expressed as

$$
\frac{G}{T}=\frac{8 \pi k(Y-1)}{\lambda^{2} S k_{1} k_{2}}
$$

where $k$ is Boltzmann's constant, $Y$ is the ratio of the power measured with the sun in the antenna mainbeam to the power measured with the antenna pointed at the cold sky, $\lambda$ is the measurement wavelength, $S$ is the solar flux density incident on the earth's atmosphere, and $k_{1}$ and $k_{2}$ are the atmospheric transmission and star shape correction factors respectively [5]. 
The atmospheric transmission factor accounts for the reduction in the received flux and is due to three atmospheric phenomena [6], gaseous attenuation, refractive spreading of the incident wavefront, and incoherent tropospheric scattering. It does not include attenuation and scattering from clouds, rain, fog, hail, or snow since the precision measurements envisioned here are assumed to be performed in clear, stable weather. If $A$ is the total zenith attenuation in decibels due to these three phenomena, then

$$
k_{1}=10^{-A / 10 \sin \theta}
$$

where $A$ is the elevation angle to the source relative to the antenna location on the earth's surface. Details of a simplified calculation for the gaseous component of $A$ are described elsewhere [7].

An algorithm derived in an earlier analysis [5] for the zenith attenuation due to the tropospheric scatter of the energy from a point source radio star and intended for the $1 \mathrm{GHz}$ to 10 $\mathrm{GHz}$ frequency range diverges above $10 \mathrm{GHz}$. Since that analysis, further measurement data [8] has come to light which provides a more detailed picture of this attenuation. The resulting curves derived from the data and which include the refractive attenuation are shown in figure 1 . The first set of data [6] were taken at $4.17 \mathrm{GHz}$ and $6.39 \mathrm{GHz}$ with a $22 \mathrm{~m}$ antenna, and the second set [8] at $15.5 \mathrm{GHz}$ and $31.6 \mathrm{GHz}$ with a $7 \mathrm{~m}$ antenna. The dashed curves through the two sets of data points are parallel, and suggest a family of straight lines for other antenna diameters. A linear interpolation in antenna diameter between the two dashed curves produced the linear portions of the curves shown. Since theory [6] indicates that the attenuation decreases with decreasing frequency, the curves were extended accordingly. When the angular diameter of the source is finite, the attenuation is reduced [6], decreasing as the ratio of source diameter to antenna beamwidth increases. The equation in figure 1 descibes this effect [5] where $d$ is the source diameter, $A_{H}$ is the half-power beamwidth, $A_{O}$ is the zenith attenuation for a point source given by the curves in the figure, and $A$ is the corresponding attenuation for the finite source diameter. An earlier investigation [5] indicates that the total error in these attenuation curves and the non-point source correction is probably in the neighborhood of $50 \%$.

The star shape correction factor accounts for variation of the solar bright ness distribution across the antenna beam pattern, and vanishes as the ratio of the angular source diameter to the antenna half-power beamwidth vanishes. In terms of a distribution $B$ and a normalized power pattern $P_{n}$,

$$
k_{2}=\frac{\int B(\Omega) P_{n}(\Omega) d \Omega}{\int B(\Omega) d \Omega}
$$

where $\Omega$ represents the two antenna pointing angles and do is the differential solid angle. The integral in the denominator is equivalent to the flux density $S$.

Sections 2 and 3 present the results of a preliminary investigation into the determination of the flux density $S$ and the star shape correction factor $k_{2}$. 


\section{Determination of the Solar Flux Density Above $5 \mathrm{GHz}$ \\ 2.1 Strategy}

Solar radio emission is the result of three superimposed sources of radiation [9], the quiet, slow, and burst components. The quiet sun emission comes from unlocalized, thermal sources in the solar atmosphere (chromosphere and corona), and is constant over periods of months to years. The slow component also has a thermal origin and originates in local sources in the vicinity of sunspots and chromospheric plagues. These partially polarized sources have periods of days, weeks, or months. The burst components are the results of thermal, synchrotron, and plasma radiation from transient sources such as flares, and are partially polarized. Their recurrence frequency and period are small for the quiescent portion of the solar cycle, increasing to two or more per a 10-hour viewing day during the active portion, which suggests the possibility of making G/T measurements using the sun without encountering a burst during the process. The fact that the quiet and slow components have periods of greater than a day further suggests the possibility of using an average of two successive daily AFGL flux density values to determine the flux at the time of the $G / T$ measurement taking place between the two AFGL measurements.

Figure 2 depicts the average solar flux density spectrum incident on the earth's atmosphere [10] with an insert [11] indicating the duration of the burst spectrum from $10 \mathrm{MHz}$ to $30 \mathrm{GHz}$. The quiet component of the solar emission dominates above $5 \mathrm{GHz}$, at which frequency most of the burst components have disappeared and the slow component is falling off. The insert shows that the duration of the remaining burst continuum is short. Thus, the solar emission above $5 \mathrm{GHz}$ is relatively stable from day to day as long as the type IV bursts are avoided, again suggesting a G/T measurement scheme using AFGL flux density values.

Superimposed on the graph of figure 2 are three sets of hashmarks representing monthly averages of AFGL flux density measurements (with atmospheric attenuation removed) [4] from January 1979 to May 1982. The measurement values approach the average quiet sun value between the "sunspot (s.s) max" and the "s.s. min" curves as the frequency increases. Thus, above the highest AFGL measurement frequency $(15.4 \mathrm{GHz})$, apparently the average of the quiet sun curves can be used to determine the flux density incident on the earth's atmosphere. Following is an approximate equation giving this average that was derived by least-square fitting the average data corresponding to the quiet sun curves in the figure and in table 1 (from reference [10]):

$$
\log S=1.20+1.10(\log f)+0.179(\log f)^{2}
$$

The flux density $S$ and the frequency $f$ are the same units as the figure. The following equations can be used to extrapolate between the AFGL measurement frequencies [12]:

$$
s=s_{1}^{c} s_{2}^{1-c}
$$

where

$$
c \equiv \frac{\ln \left(f / f_{1}\right)}{\ln \left(f_{2} / f_{1}\right)} .
$$

$S$ has the same units $\left(W / M^{2} / H z\right)$ as the graph, and $f_{1}$ and $f_{2}$ correspond to $S_{1}$ and $S_{2}$, 
respectively. The subscripts denote the two AFGL values of flux density and frequency between which the flux density $S$ at frequency $f$ is desired.

The preceding discussion suggests the following strategy for determining the solar flux density above $5 \mathrm{GHz}$ : 1) if the $\mathrm{G} / \mathrm{T}$ measurement frequency is $4.995 \mathrm{GHz}, 8.8 \mathrm{GHz}$, or $15.4 \mathrm{GHz}$, use an average of two adjacent daily AFGL flux values; 2) if the measurement frequency is between two AFGL frequencies, use the average determined in 1) for both AFGL frequencies and translate the result to the desired frequency via eqs (5) and (6); and 3) if the frequency is above $15.4 \mathrm{GHz}$, use eq (4) to determine the flux density. Since the reported AFGL flux density values are corrected for bursts, it is only then necessary to insure that the G/T measurement did not take place during a burst.

To illustrate, suppose a series $10 \mathrm{GHz}$ G/T measurements were performed in Boulder, Colorado, at various times between $7 \mathrm{a.m}$. (1400 u.t. (universal time)) and 5 p.m. (2400 u.t. local standard time on October 17, 18, 19, 1983. The appropriate AFGL flux density values (in units of 10-22 $\mathrm{W} / \mathrm{m} / \mathrm{Hz}$ ) that bracket $10 \mathrm{GHz}$ on these days are isolated in the box of figure 3 for the month in question [4]. The " $\mathrm{C}$ " value from eq (6) for the frequencies indicated $(8.8 \mathrm{GHz}, 10 \mathrm{GHz}, 15.4 \mathrm{GHz})$ turns out to be 0.7716 (with $f_{1}=8.8, f=10$, and $f_{2}=15.4$ ). Using this value, the flux densities in the box, and eq (5), the $10 \mathrm{GHz}$ flux density values at the AFGL measurement time (approximately 1630 u.t.) on the 17th, 18th, 19th, and the 20th are 334, 321, 330, and 326, respectively. Linear interpolation between any appropriate two of these values will then yield the correct flux value for a specific Boulder G/T measurement time. Figure 4 is a reproduction of another page from the "Solar-Geophysical Data" report that indicates the time and size (in flux density units) of any bursts that have taken place during the days in which the G/T measurements were being performed. The data in the boxes show the larger microwave bursts taking place during the $G / T$ measurement times in question (1400 u.t. to 2400 u.t.), and thus any $G / T$ measurements performed during these bursts chould be discarded. The sinaller bursts shown in the figure, al though somewhat arbitrarily excluded, have a less significant effect on the $G / T$ measurement.

\subsection{Flux Density}

For simp?icity the preceding illustration ignores atmospheric attenuation and the true earthsun distance at the time of the $G / T$ measurements. A complete flux density determination between AFGL frequencies prorseds as follows: 1) calculate the flux densities $S_{1}$ and $S_{2}$ corresponding to the appropriate two AFGL frequencies for the $G / T$ measurement time between the two AFGL daily measurements; 2) adjust $S_{1}$ and $S_{2}$ for the true earth-sun distance (the values reported in figure 3 are normalized to 1 astronorical unit); 3) remove the effects of atmospheric attenua *ion from $S_{1}$ and $S_{2}$ via eq (2); 4) use eqs (5) and (6) to obtain the flux density $S$ incident on tir - in's atmosphere at the $G / T$ measurement frequency; and 5) apply eq (2) a second time at the measurement frequency to obtain the flux density at the location on the earth's surface where the $G / T$ measurement is performed. Above $15.4 \mathrm{GHz}$ eq (4) can be used to estimate the flux density incident on the atmosphere, after which step 5 is applied.

Prior to, but not afterward, January 1980, AFGL removed the effects of atmospheric attenuation from their measurements and reported flux density values incident on the atmosphere. Therefore, the flux density values reported in figure 3 contain the atmospheric attenuation. 
To illustrate, suppose a G/T measurement is performed at an antenna site in Boulder on October 17, 1983, as the sun transits the local meridian (1848 u.t. [13]). The AFGL flux density values measured earlier on the 17 th and on the following day during meridian transit (1630 u.t. [13]) at the Sagamore Hill observatory are reported (fig. 3) as 282 and 268 at $8.8 \mathrm{GHz}$, and as 588 and 587 at $15.4 \mathrm{GHz}$. By a linear extrapolation in time between these daily measurements, flux density values at $1848 \mathrm{u} . t$. of 280.6 and 587.9 are obtained for $8.8 \mathrm{GHz}$ and $15.4 \mathrm{GHz}$, respectively. The true earth-sun distance (in units of 1 astronomical unit) at $1848 \mathrm{u} . t$. on the 17 th was 0.996 [13]. Thus, the true flux density values at the G/T measurement time are a factor of $1.008\left(0.996^{-2}\right)$ larger than the values reported by AFGL, or 282.8 and 592.6 for $8.8 \mathrm{GHz}$ and $15.4 \mathrm{GHz}$, respectively.

The average solar declination during the AFGL and G/T measurements was a negative 9.4 degrees [13]. Combined with the Sagamore Hill latitude of $40.6^{\circ}$ north, this leads to a solar elevation angle at that site of $38.0^{\circ}$. The zenith values for the gaseous component of the atmospheric attenuation in October at the observatory are [7] $0.042 \mathrm{~dB}$ and $0.080 \mathrm{~dB}$ for $8.8 \mathrm{GHz}$ and $15.4 \mathrm{GHz}$, respectively. The zenith values for the diffusive and refractive attenuation for the average solar diameter of $0.58^{\circ}$ [13] during the measurements are (fig. 1) $0.012 \mathrm{~dB}$ at $8.8 \mathrm{GHz}$ and $0.015 \mathrm{~dB}$ at $15.4 \mathrm{GHz}$. Combining these with the gaseous components gives a total zenith attenuation of $0.054 \mathrm{~dB}$ at $8.8 \mathrm{GHz}$ and $0.095 \mathrm{~dB}$ at $15.4 \mathrm{GHz}$. These latter values with the $38.0^{\circ}$ declination 1 ead via eq (2) to atmospheric correction factors of 0.980 at $8.8 \mathrm{GHz}$ and 0.965 at $15.4 \mathrm{GHz}$. Dividing the previous flux density values by these correction factors leads to 288.6 and 614.1 at 8.8 GHz and $15.4 \mathrm{GHz}$, respectively, for the flux densities incident on the atmosphere. Equations (5) and (6) can now be used to obtain an estimate of the $10 \mathrm{GHz}$ flux density and results in a value 342.9. If the Boulder site is at $40^{\circ}$ north latitude, the solar elevation angle there is $40.6^{\circ}$. Combining a gaseous attenuation of $0.029 \mathrm{~dB}$ [7] with a diffusive and refractive attenuation of $0.011 \mathrm{~dB}$ (fig. 1) yields a correction factor of 0.986 for a $40.6^{\circ}$ elevation angle, resulting in a value of 338.1 for the flux density at the Boulder site.

\subsection{Errors}

The total error in determining the flux density from the AFGL measurements is the sum of four parts: the AFGL measurement error; the error in using eqs (5) and (6) to extrapolate between AFGL frequencies; the error in estimating the atmospheric transmission factor; and the error in estimating the flux density by the linear time interpolation between the daily AFGL measurements. This latter error for frequencies above the highest AFGL frequency (15.4 GHz) is at present taken as the maximum error (halfway) between the highest AFGL frequencies, 8.8 GHz and $15.4 \mathrm{GHz}$.

The AFGL error for measuring the flux density is $5 \%$ [12].

An error of $1 \%$ is assigned to the uncertainty in extrapolating between AFGL frequencies, and was determined by comparing the $8.8 \mathrm{GHz}$ average value (average of the AFGL hashmarks in figure 2 ) with the $8.8 \mathrm{GHz}$ value obtained via eqs (5) and (6) and the average AFGL values at $4.995 \mathrm{GHz}$ and $15.4 \mathrm{GHz}$.

The total (gaseous + diffusive + refractive) zenith attenuations for Boulder at $10 \mathrm{GHz}$ and Sagamore $\mathrm{Hill}$ at $8.8 \mathrm{GHz}$ and $15.4 \mathrm{GHz}$ are $0.040 \mathrm{~dB}, 0.054 \mathrm{~dB}$, and $0.095 \mathrm{~dB}$, respectively. All of these values are uncertain by $50 \%$ [5], leading to transmission coefficient errors of $0.7 \%$, $1.0 \%$. 
and $1.8 \%$, respectively. The overall transmission coefficient error is a weighted sum $(0.7+c \times 1.0+(1-c) \times 1.8)$ of these separate errors with a value of approximately $2 \%$ for the value of $c$ determined in section 2.1 .

Figure 5 is a plot of a number of hypothetical smoothed flux density curves between the AFGL values of $S\left(t_{1}\right)$ and $S\left(t_{2}\right)$ measured during meridian passage at $t$ ime $t_{1}$ and time $t_{2}$ the following day $\left(t_{2} \doteq t_{1}+24\right.$ hours). The linear curve whose equation is shown in the figure was previously used to estimate the flux density at time $t$ between the AFGL measurement times $t_{1}$ and $t_{2}$, where $h$ is the relative forward interval between $t_{1}$ and $t . s(t)$ is the flux density at $t$ for the true (but unknown) curve, and $\sigma_{S}$ is the estimated standard deviation of the possible values at. $t$ for the probability density curve shown. The forward relative interval $h$ is defined in the figure. $\Delta S$ is defined to be the difference between the linear approximation and the unknown flux density $S(t)$, and is therefore a random variable whose estimated standard deviation $\sigma_{\Delta}$ is a measure of the error in using the linear approximation. A simple analysis of the situation depicted in the figure shows that the two standard deviations are related by the equation

$$
\sigma_{\Delta}^{2}=2 \sigma_{s}^{2}\left[1-h+h^{2}+h(1-h) \rho_{1}-(1-h) \rho_{1}^{h}-h \rho_{1}^{1-h}\right]
$$

where $\rho_{1}$ is the estimated autocorrelation function [14] between measured flux density values 24 hours $\left(\stackrel{\rho}{=} t_{2}-t_{1}\right)$ apart. $\rho_{1}^{h}$ and $\rho_{1}^{1-h}$ are, respectively, approximations to the autocorrelation functions betiveen flux density values $t-t_{1}$ and $t_{2}-t$ apart. The flux-density variations are assumed to be sufficiently stationary for eq (7) to give some indication of the time interpolation error.

Figure 6 shows a plot of the $8.8 \mathrm{GHz}$ AFGL flux density values measured from January through June 1979. The change of levels at L1, L2, and L3, and the slopes at S1, S2, and S3 indicate that the variation is not stationary and should possibly be modeled [14] as an ARIMA ( $p, 1, q$ ) or an $\operatorname{ARIMA}(p, 2, q)$ process to estimate the standard deviation of the residual day-to-day fluctuations of interest. However, for a preliminary (and more conservative) estimate of $\sigma_{S}$, the data shown was assumed to be stationary, resulting in a value of 24 for $\sigma_{S}$ and a value of 0.9 for $\rho_{1}$. With these values eq (7) is approximated by

$$
\sigma_{\Delta} \approx 11\left(h-h^{2}\right)^{1 / 2}
$$

This equation shows a maximum of 5.5 halfway between $t_{1}$ and $t_{2}$, and is assumed to hold for the entire range from $5 \mathrm{GHz}$ to $15.4 \mathrm{GHz}$. For the illustration in section 2.2 , $h$ has the value of 0.096 ((18.8-16.5)/24), yielding an estimated standard deviation from eq (8) of 3.2 or $1 \%$ $(3.2 / 337)$, or a $3 \sigma$ error of approximately $3 \%$.

Collecting the previous errors leads to a total error of $11 \%(3 \sigma)$ in determining the solar flux density for the illustration presented in section 2.2. The error at lower elevation angles and/or higher frequencies will be greater, primarily because of increased gaseous attenuation.

\section{Star Shape Correction Factor}

For antenna half-power beamwidths (HPBW) greater than about five angular source diameters, examination of eq (9) shows that ratio in eq (3) can be replaced by unity with an error less than 
1\%. To use the celestial-source method of measuring G/T accurately below five source diameters, however, an estimate of the variation of the source brightness distribution across the antenna inainbeam indicated in the numerator of eq (3) must be made. For sources with stable and predictable distributions and Gaussian mainbeams, this can be accomplished by replacing the ratio in eq (3) by a simple formula depending only upon the ratio of HPBW to source diameter. Furthermore, if the source diameter is properly chosen, the formula will provide an accurate estimate of the correction factor for any HPBW/diameter ratio above unity [15,2]. As wi11 be seen there is reason to expect that this technique will also work for the sun, even though the solar brightness distribution is continuously changing. Errors in the star shape correction factor have not yet been investigated.

For a source resembling a circular disk and a Gaussian mainbeam, eq (3) 1eads to

$$
k_{2}=\frac{1-e^{-x^{2}}}{x^{2}}
$$

where

$$
x^{2} \equiv\left(\frac{d}{\theta_{H}}\right)^{2} \ln 2 .
$$

$d$ is the effective rf angular diameter of the source, and $\theta_{H}$ is the antenna HPBW. The diameter is chosen to minimize the difference

$$
\frac{1-e^{-x^{2}}}{x^{2}}-\frac{\int B(\Omega) P_{n}(\Omega) d \Omega}{\int B(\Omega) d \Omega}
$$

for $\theta_{H} / d$ greater than unity. The same expressions (eqs (9) and (10)) hold for an elliptical disk with $d^{2}$ replaced by the product of the major and minor source diameters. No significant reduction in the difference in eq (10) is achieved with this latter model, however, even though the solar brightness distribution shows a decided ellipticity (fig. 7, [9]).

Figure 2 shows that, except for the burst components which are assumed to be missing during the G/T measurement, the quiet sun emission plays an increasingly dominant role in the total emission above $5 \mathrm{GHz}$ where the slow component begins to fall off. Furthermore, observations of the solar brightness distribution in the decimeter, centimeter, and millimeter wave frequency regions reveal that, although the effective brightness temperature varies with the solar cycle, the distributions remain relatively constant [9]. It is this constant part of the brightness distribution that will presumably be amenable to the technique of the previous paragraph, with fluctuations in the result coming from the more variable slow emission component. The degree of success will depend upon how minor these fluctuations turn out to be, but hopefully at least some accuracy in $k_{2}$ will be gained below five source diameters. This question can only be decided after a number of distributions at various frequencies and at various times during the solar cycle have been convoluted with the antenna beam pattern. To date this $h$ as not been done.

Since the effective diameter or diameters for the solar distributions have not yet been determined, the question of what diameter $d$ to use in eq (10) remains. A glance at figure 7 shows 
that the polar profiles tend to average out as a constant up to the optical diameter, with the equatorial profile averages extending somewhat beyond. This situation implies that the effective rf diameter will extend beyond the optical diameter, which in turn will depend upon the results from convolving the brightness distributions with the Gaussian beam pattern. Based on a circular disk model of the quiet-sun atmosphere with a constant chromospheric temperature of $3 \times 10^{4}$ kelvins and a coronal temperature of $10^{6}$ kelvins, Smerd [16] calculated a brightness temperature distribution and compared it to the apparent brightness temperature derived from the same model. The apparent temperature is defined as the temperature of a blackbody having the same diameter as the optical disk and radiating the same apparent flux density. The results are shown in figure 8 where the ratio of two particular brightness temperature contours to the apparent temperature are plotted. The upper curve is where the contour temperature falls to $1 \%$ of the apparent temperature and the lower curve is where it falls to $10 \%$. As expected the ratios approach unity with increasing frequency as the origin of the radiation is increasingly confined to the chromosphere. The dashed line approximating the $10 \%$ curve and ending at $30.3 \mathrm{GHz}$ will be used temporarily to define the effective diameter for eq (10). Thus,

$$
d=d_{0}\left\{\begin{array}{cc}
1.240-0.162 & \log f, \\
1 & , f \leqslant f \leqslant 30.3 \\
& f \geqslant 30.3
\end{array}\right.
$$

where $d_{0}$ is the optical diameter obtained from the Astronomical Almanac [13] at the time of the $\mathrm{G} / \mathrm{T}$ measurement. For the illustration in section 2.2, the Boulder antenna diameter is $4.3 \mathrm{~m}$ ( $\mathrm{fig}$. 1) yielding a $10 \mathrm{GHz} \mathrm{HPBW}$ of $0.49^{\circ}$. At the measurement time the optical solar diameter was $0.54^{\circ}$ [13] which yields an $r f$ diameter via eq (12) of $0.58^{\circ}$. Equations (9) and (10) then give a star shape correction factor of 0.64 .

\section{Discussion and Conclusions}

The curves in figure 1 used for determining the diffusive and refractive attenuation $\mathrm{fc}^{\mathrm{n}}$ a point source, although quite rough and incomplete, offer an improvement over the previous algorithm [5] that diverges above $10 \mathrm{GHz}$.

The total error in estimating the solar flux density from $5 \mathrm{GHz}$ to $15.4 \mathrm{GHz}$ in clear, stable, weather is $11 \%$, and is the sum of $5 \%$ for the AFGL measurements, $1 \%$ for extrapolating between two AFGL frequencies, 3\% for estimating the flux density between the daily AFGL measurement times, $1.3 \%$ for removing the atmospheric attenuation from the AFLG measurements, and $0.7 \%$ for estimating the atmospheric attenuation at the G/T measurement frequency. Discarding the $0.7 \%$ error, the sum comes to about $8 \%$, and represents the error in determining the flux density incident on the earth's atmosphere. Since the average of the AFGL hashmarks in figure 2 approaches the area between the "s.s. max" and "s.s. min" curves as the frequency increases, and since their spread and that between the curves decreases with frequency, it appears that an $8 \%$ error in using eq (4) to determine the solar spectrum above $15.4 \mathrm{GHz}$ incident on the earth's atmosphere is reasonable for the spectrum shown in the figure.

The brightness distribution of the quiet sun above $5 \mathrm{GHz}$ is relatively insensitive to the solar cycle. This, coupled with the fact that the quiet-sun component also dominates above this frequency, implies the possibility of finding an effective $r f$ diameter (as a function of frequency) that minimizes the error in using the circular disk approximation to the star shape 
correction factor in eq (3), improving G/T measurement accuracy for HPBW/source-diameter ratios between one and five.

Although the preceding conclusions appear to be reasonable, there still remains a considerable amount of work to be completed before final acceptance.

The majority of the work described in this report was carried out in 1979 and was part of a larger effort to design and construct an earth terminal measurement system capable of precision antenna system measurements. Therefore, much of the direction and inspiration for this work came from other individuals associated with the project, principally David Wait and John Wakefield.

\section{References}

[1] Wait, D. F. Precision measurements of antenna system noise using radio stars. IEEE Trans. on I\&M, Vo1. IM-32, No. 1: 110-116; 1983 March.

[2] Daywitt, W. C. An error analysis for the use of presently available lunar radio flux data in broadbeam antenna-system measurements. Nat. Bur. Stand. (U.S.) Tech. Note 1073; 1984 February.

[3] Wait, D. F.; Daywitt, W. C. Preliminary examination of $20 \mathrm{GHz} G / T$ measurements of earth termina1s. Nat. Bur. Stand. (U.S.) Int. Rpt. 83-1686; 1983 March.

[4] Solar-Geophysical Data; prompt reports. Ed. H. E. Coffey; No. 470, Part 1, 1983 November.

[5] Daywitt, W. C. Error equations used in the NBS precision G/T measurement system. Nat. Bur. Stand. (U.S.) Int. Rpt. 76-842, 1976 September.

[6] Yokoi, H.; Yamada, M.; Satoh, T. Atmospheric attenuation and scintillation of microwaves from outer space. Publ. Astr. Soc. Japan, Vol. 22, No. 4, 511-524; 1970.

[7] Daywitt, W. C. Atmospheric propagation equations used in the NBS earth terminal measurement system. Nat. Bur. Stand. Int. Rpt. 78-883, 1978 April.

[8] Yokoi, H.; Yamada, M. Measurements of earth-space propagation characteristics at $15.5 \mathrm{GHz}$ and $31.6 \mathrm{GHz}$ using celestial radio sources. Trans. IECE 174/2, Vol. 57-8, No. 2; 121-128; 1974 February.

[9] Kundu, M. R. Solar Radio Astronomy. New York, N.Y.: Interscience Publishers; 1965.

[10] Allen, C. W. Astrophysical Quantities. London: The Athlone Press/University of London: 1976. 
[11] Mangis, S. J. Introduction to solar-terrestrial phenomena and the space environment services center. NOAA Tech. Rpt. ERL 315-SEL 32, 1975 January.

[12] Guidice, D. A.; Castelli, J. P. The use of extraterrestrial radio sources in the measurement of antenna parameters. IEEE Trans. Aerospace and Electronic Systems: Vol. AES-7, No. 2; 226-234; 1971 March.

[13] The Astronomical Almanac for the Year 1983. Washington: U.S. Government Printing Office.

[14] Box, G. E. P.; Jenkins, G. M. Time Series Analysis--Forecasting and Control. San Francisco: Holden-Day; 1976.

[15] Kanda, M. An error analysis for absolute flux density measurements of Cassiopeia A. IEEE Trans. on I\&M, Vol. IM-25, No. 3; 173-182; 1976 September.

[16] Smerd, S. F. Radio-frequency radiation from the quiet Sun. Aus. J. Sci. Res., A3, 34; 34$59 ; 1950$. 


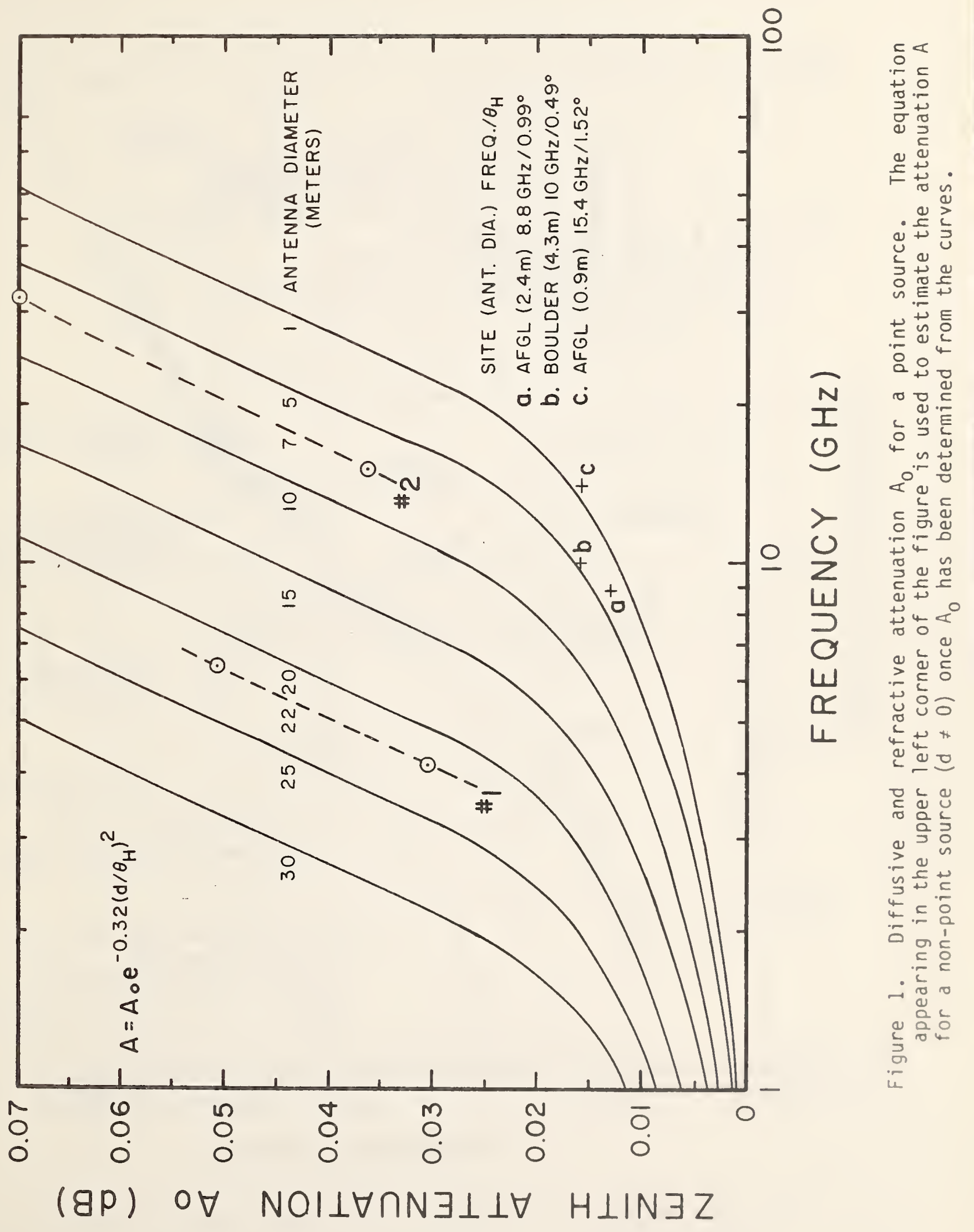




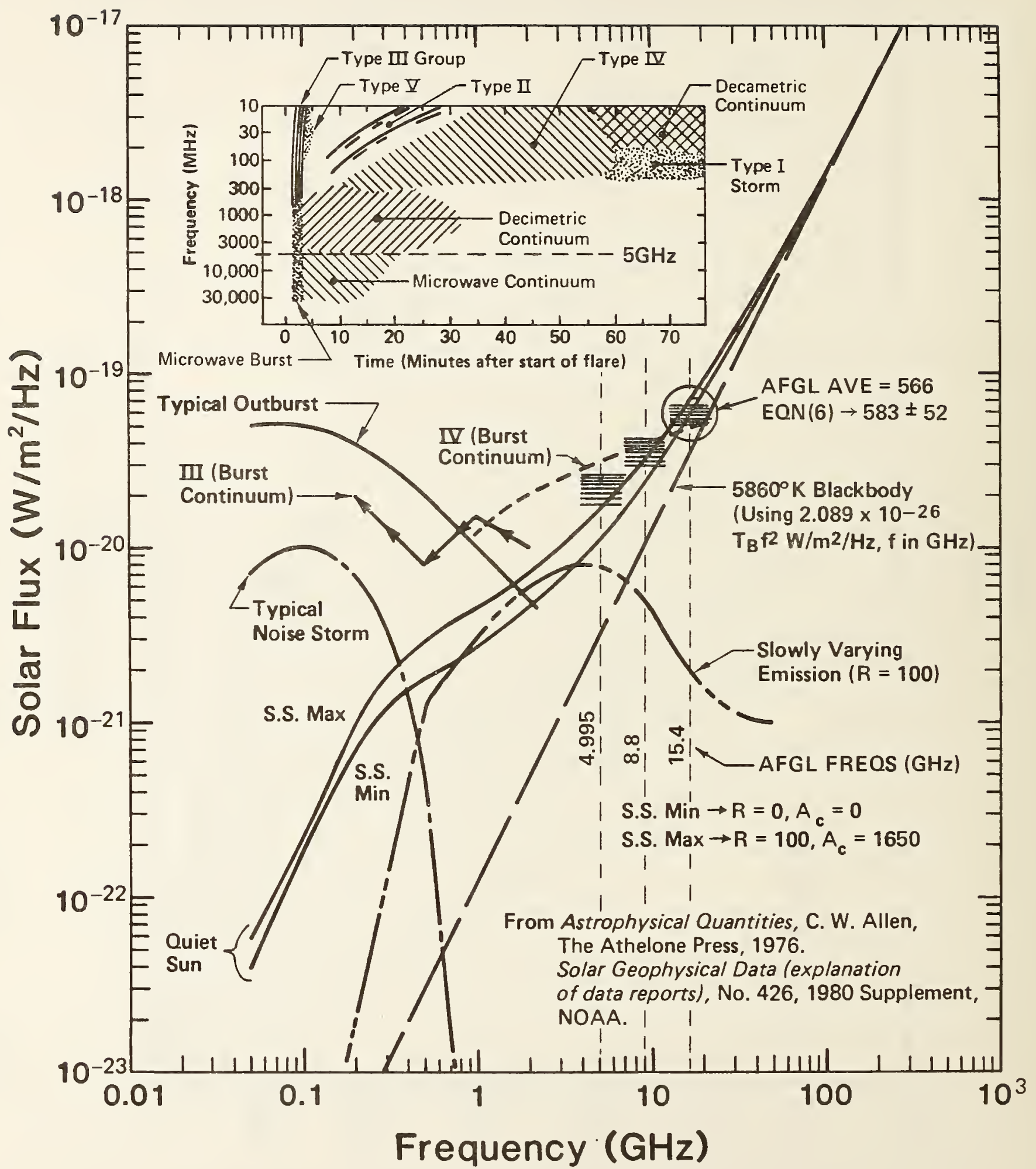

Figure 2. Solar radio spectrum. (The symbols are explained more fully in reference [10].) 
DAILY SOLAR INDICES

$\operatorname{cct}^{9} 83$

OCTOBER 1983

\begin{tabular}{|c|c|c|c|c|c|c|c|c|c|c|c|c|c|c|}
\hline Doy & $\begin{array}{l}\text { ulian } \\
\text { Day }\end{array}$ & $\begin{array}{c}8 \text { artels } \\
\text { Cycle } \\
\text { Day }\end{array}$ & $\begin{array}{l}\text { Sun } \\
\text { Num } \\
R_{1}\end{array}$ & $\begin{array}{l}\text { spot } \\
\text { pers } \\
R_{A} \text {. }\end{array}$ & $\begin{array}{c}\text { Oos Flux } \\
\text { Ottawa } \\
(2800)\end{array}$ & $\begin{array}{r}\text { SQMR } \\
(15400)\end{array}$ & $\begin{array}{l}\text { - Solar } \\
\text { SGMR } \\
(8800)\end{array}$ & $\begin{array}{l}\text { FI ux } A C \\
\text { SGMR } \\
(4995)\end{array}$ & $\begin{array}{l}\text { djusted } \\
\text { Ottawa } \\
(2800)\end{array}$ & $\begin{array}{l}\text { to } 1 \text {. A } \\
\text { SGNR } \\
(2695)\end{array}$ & $\begin{array}{c}\text { stronom i } \\
\text { SGMR } \\
\text { (1415) }\end{array}$ & $\begin{array}{l}\text { ical Un } \\
\text { SGMR } \\
(606)\end{array}$ & $\begin{array}{r}1+-- \\
\text { SGMR } \\
(410)\end{array}$ & $\begin{array}{l}\text { Sind } \\
(245)\end{array}$ \\
\hline 01 & 275 & 11 & 32 & 33 & 117.3 & 565 & 266 & 148 & 117.5 & 105 & 97 & 79 & 37 & 22 \\
\hline 02 & 276 & 12 & 51 & 57 & 120.3 & 566 & 266 & 152 & 120.4 & 106 & 97 & 75 & 34 & 19 \\
\hline 03 & 277 & 13 & 63 & 69 & 123.0 & -- & $\cdots$ & -.. & 123.1 & $-\cdot$ & -- & -- & -- & -- \\
\hline 04 & 278 & 14 & 74 & 73 & 125.1 & 585 & 271 & 156 & 125.1 & 110 & 99 & 76 & 33 & 15 \\
\hline 05 & 279 & 15 & 65 & 59 & $126.6^{*}$ & 586 & 286 & 160 & $126.6^{*}$ & 121 & 103 & 80 & 33 & 23 \\
\hline 06 & 280 & 16 & 75 & 76 & 132.8 & 592 & 277 & 161 & 132.7 & 127 & 106 & 82 & 34 & 17 \\
\hline 07 & 281 & 17 & 87 & 86 & 134.0 & 596 & 281 & 158 & 133.9 & 103 & 109 & 87 & 36 , & 19 \\
\hline 08 & 282 & 18 & 99 & 89 & $131.4 *$ & 582 & 280 & 157 & $131.1 *$ & . 113 & 111 & 87 & $36^{\prime}$ & 22 \\
\hline 09 & 283 & 19 & 106 & 100 & 130.8 & 580 & 276 & 156 & 130.4 & 109 & 112 & 85 & 39 & 36 \\
\hline 10 & 284 & 20 & 108 & 113 & $134.0^{*}$ & 601 & 277 & 160 & $133.6^{*}$ & 115 & 119 & 84 & 35 & 24 \\
\hline 11 & 285 & 21 & 130 & 134 & 138.9 & 593 & 271 & 164 & 138.3 & 121 & 118 & 83 & 37 & 19 \\
\hline 12 & 286 & 22 & 122 & 114 & 134. & 570 & 265 & 157 & 133.7 & 110 & 113 & 73 & 32 & 16 \\
\hline 13 & 287 & 23 & 100 & 94 & $134.2^{*}$ & 554 & 296 & 173 & $133.5^{*}$ & $\quad 121$ & 114 & 87 & 37 & 15 \\
\hline$i 4$ & 288 & 24 & 75 & 73 & $132.2^{*}$ & 618 & 299 & 175 & $131.5 *$ & 119 & 110 & 82 & $=5$ & 16 \\
\hline 15 & 289 & 25 & 72 & 64 & 127.8 & --- & --- & --- & 127.0 & --- & -.- & -- & -- & -- \\
\hline 16 & 290 & 26 & 61 & 55 & $118.0 \mathrm{~A}$ & 605 & 284 & 161 & $117.2 \mathrm{~A}$ & 101 & 99 & 75 & 32 & 16 \\
\hline 17 & 291 & 27 & 60 & 53 & $111.7^{*}$ & 588 & 282 & 152 & $110.9 *$ & 97 & 95 & 76 & 35 & 20 \\
\hline 18 & 292 & 1 & 63 & 57 & 104.4 & 587 & 268 & 138 & 103.6 & 89 & 86 & 68 & 31 & 17 \\
\hline 19 & 293 & 2 & 46 & 36 & 106.0 & 591 & 278 & 142 & 105.2 & 93 & 89 & 73 & 33 & 15 \\
\hline 20 & 294 & 3 & 26 & 24 & 100.0 & 539 & 274 & 132 & 99.1 & 83 & 87 & 72 & 31 & 15 \\
\hline 21 & 295 & 4 & 18 & 18 & 90.1 & 589 & 265 & 125 & 89.3 & 79 & 84 & 69 & 30 & 15 \\
\hline 22 & 296 & 5 & 22 & 25 & 88.1 & 580 & 256 & 121 & 87.2 & 79 & 77 & 71 & 30 & 13 \\
\hline 23 & 297 & 6 & 22 & 16 & 88.7 & 565 & 257 & 123 & 87.8 & 75 & 79 & 70 & 31 & 18 \\
\hline 24 & 298 & 7 & 20 & 19 & 89.6 & 536 & 246 & 121 & 88.6 & 73 & 79 & 72 & 30 & 14 \\
\hline 25 & 299 & 8 & 18 & 17 & 90.3 & 566 & 254 & 122 & 89.2 & 81 & 84 & 70 & 28 & 13 \\
\hline 26 & 300 & و & 22 & 13 & 90. & 579 & 262 & 126 & 89.1 & 80 & 79 & 73 & 30 & 14 \\
\hline 27 & 301 & 10 & 12 & 13 & 90.1 & 575 & 255 & 126 & 88.9 & 83 & 80 & -- & 31 & 14 \\
\hline 28 & 302 & 11 & 11 & 12 & 91.6 & 578 & 256 & 124 & 90.4 & 84 & 79 & 74 & 34 & 15 \\
\hline 29 & 303 & 12 & 16 & 0 & 92.0 & 571 & 259 & 124 & 90.7 & 78 & 76 & 68 & 29 & 14 \\
\hline 30 & 304 & 13 & 15 & 8 & 93.9 & 565 & 252 & 124 & 92.6 & 82 & 80 & 50 & 26 & 14 \\
\hline 31 & 305 & 14 & 19 & 15 & $97.0^{*}$ & 585 & 262 & 128 & $95.5^{*}$ & 86 & 83 & 73 & 28 & 15 \\
\hline Mear & & & 55 & 52 & 112.4 & 581 & 270 & 143 & 111.7 & 97 & 95 & 76 & 32 & 17 \\
\hline
\end{tabular}

Adjusted for burst in progress at time of measurement.

The observed and the gdjusted Ottawa fluxes tabulated bove ore the "Serles C" dally values reported by the Algonquin Radlo Observatory, Ottawa. Ontarlo, Canada. The letter "A" following an entry designates an Interpolated flux. Numbers in parentheses in the column headings denote frequencies in lyz.

Equipment problems produced the gaps shown here In the Alr weather Service's Sagamore Hill (SGMS) Observations.

The Internatlonal and American sunspot numbers shown above are prellminary values.

Figure 3. Smoothed solar flux density (in units of $\mathrm{W} / \mathrm{m}^{2} / \mathrm{Hz}$ ) measured daily by the AFGL Sagamore Hill observatory (SGMR) at approximately solar meridian pass age each day and adjusted to one astronomical unit. The box contains the flux densities of interest. 
SOLAR RADIOEMISSION

OCTOBER 1983

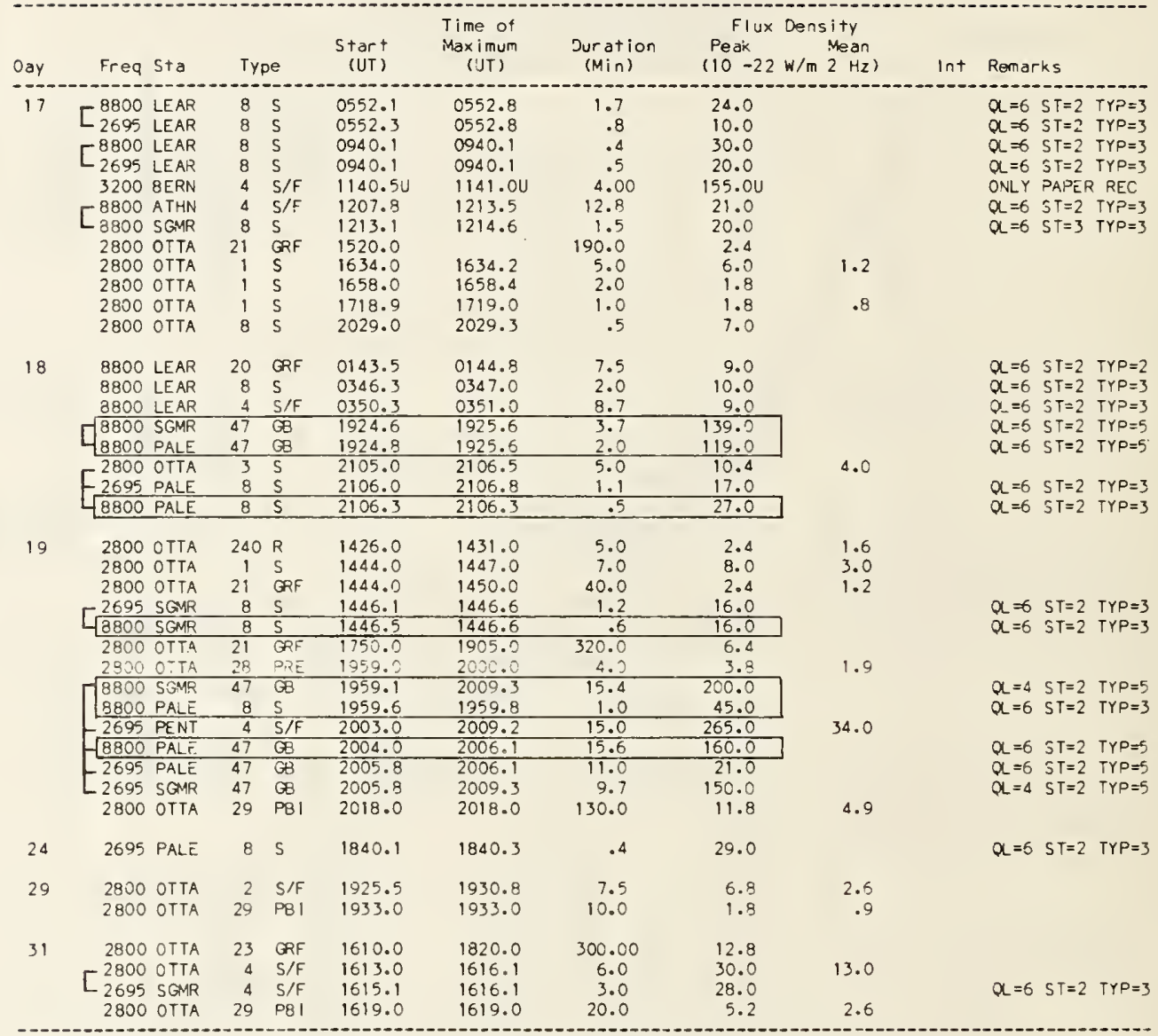

Reports are recelved rout Inely from the following observatorles:

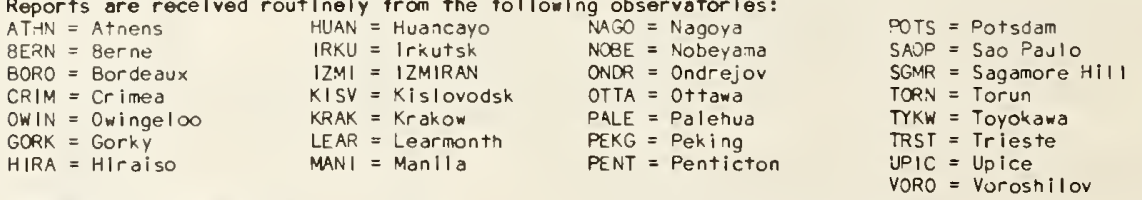

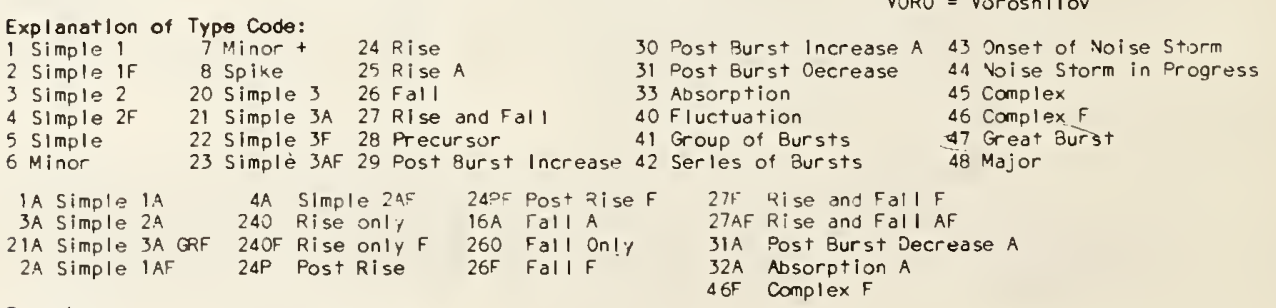

Remarks:

$Q L=$ Quality (1=poor to $6=$ excellent $)$

ST = Status ( $1=$ real time; $2=$ final; $3=$ correction; $4=$ deletion

TYP = Type $11=$ noise storm; $2=r$ Ise in base level; $3=$ minor; $4=$ group; $5=$ major; $6=$ major plus; $7=$ Castel 11 U-type burst

Figure 4. Selected, short duration, radio emissions. Major events to be avoided during the $G / T$ measurement days are contained in the boxes. 


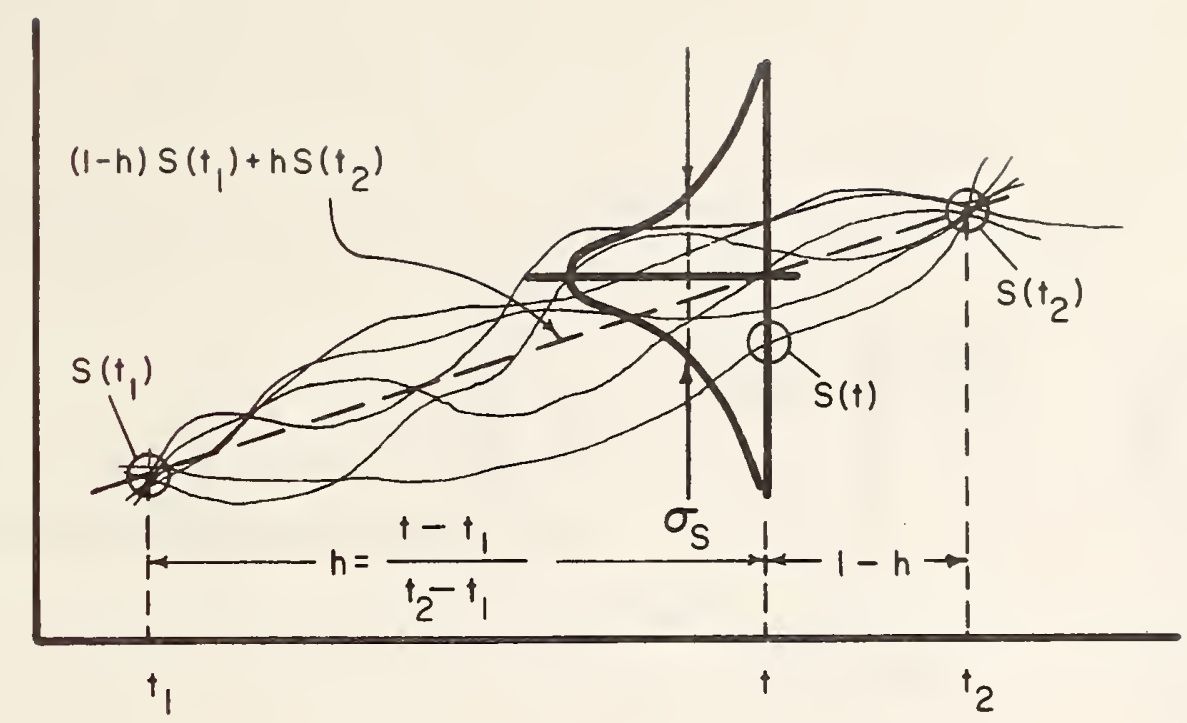

Figure 5. Hypothetical flux density curves.

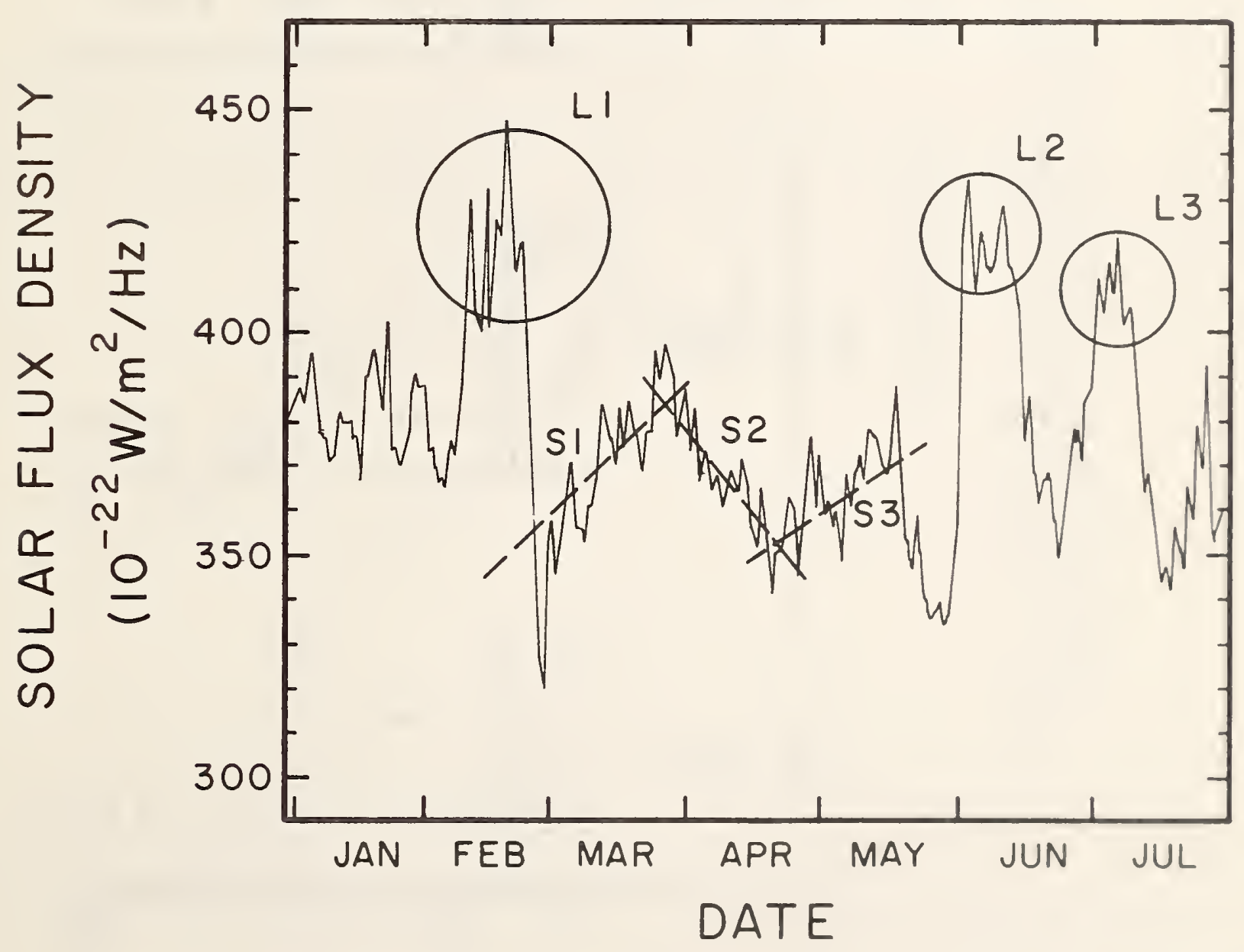

Figure 6. Smoothed solar flux density at $8.8 \mathrm{GHz}$ measured by AFGL from January to August 1979. 

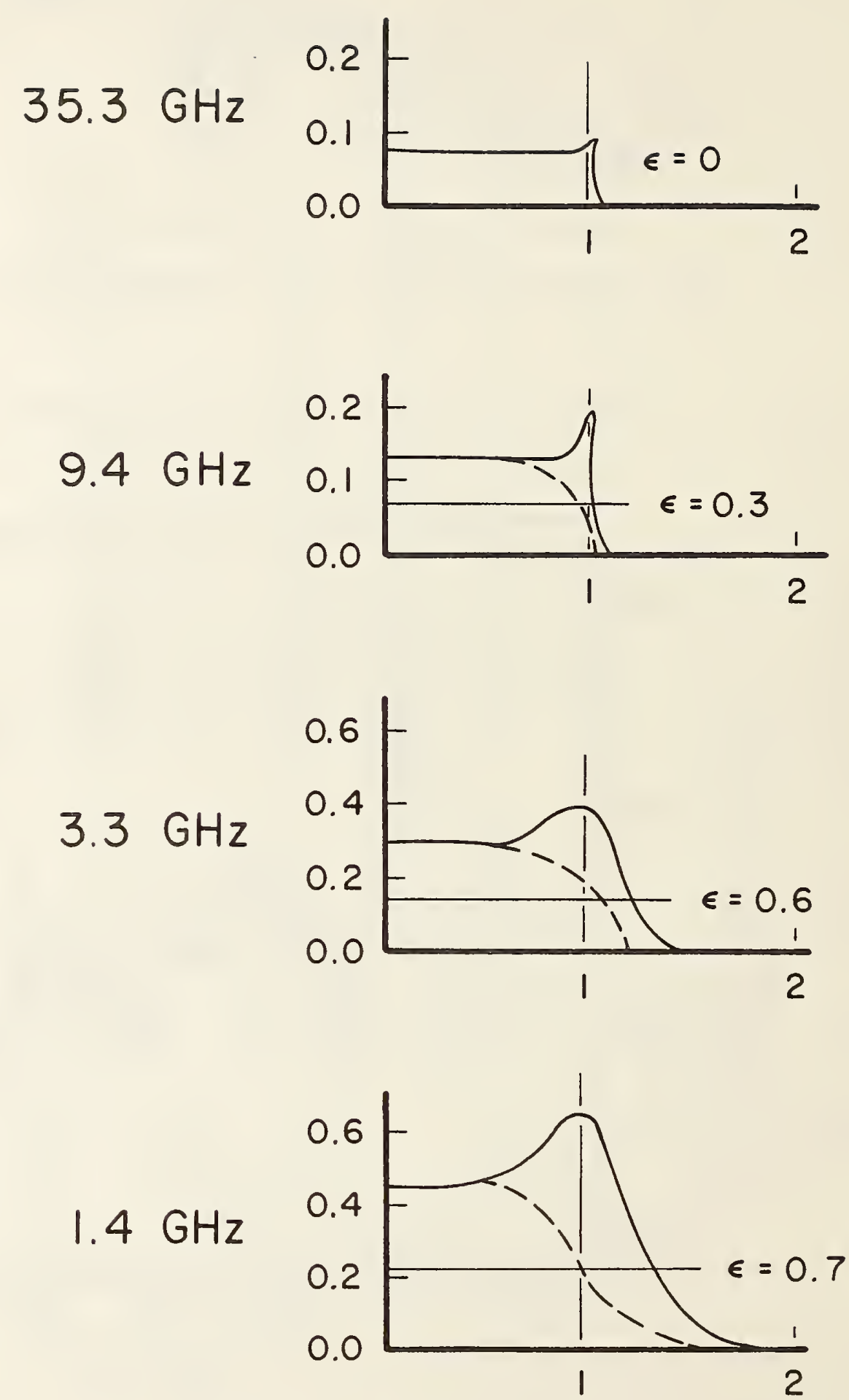

Figure 7. Quiet-sun brightness temperature profiles (in units of $10^{5}$ kel vins) at various frequencies. The solid curves are equatorial profiles, and the dashed curves are polar profiles. The values of $\varepsilon$ represent the ellipticity at the half-power levels indicated. The abscissa is in units of the optical solar radius. (After Kundu [9].) 


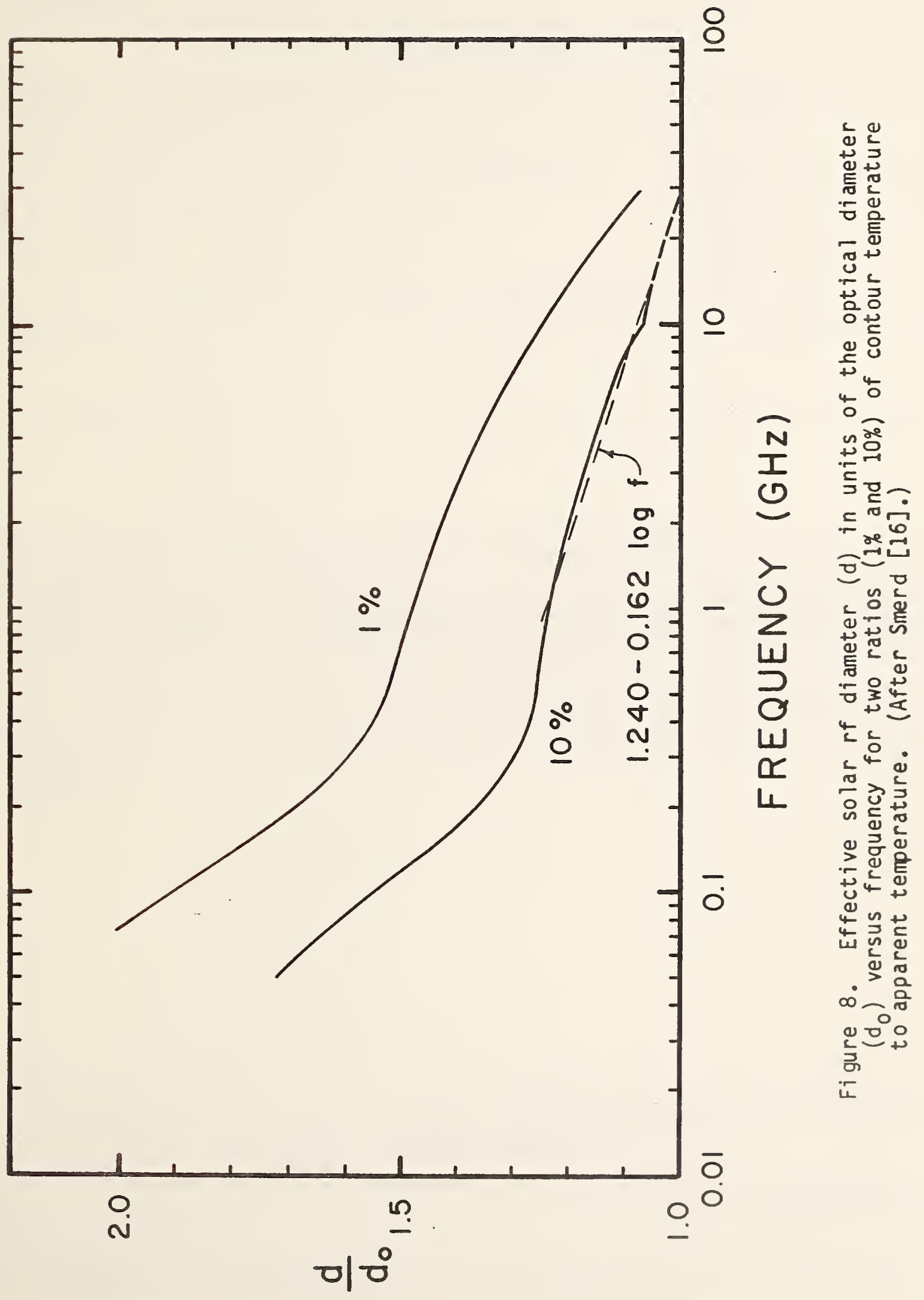


Table 1. Average flux density $S\left(W / \mathrm{m}^{2} / \mathrm{Hz}\right)$ versus frequency for the quiet sun radiation in terms of the base-10 logarithm of $S$. (From Allen [10].)

QUIET SUN RADIATION

FREQUENCY (GHz) LOg $S$

$\begin{array}{rl}10 & 2.48 \\ 20 & 2.93 \\ 50 & 3.57 \\ 100 & 4.125 \\ 200 & 4.69 \\ 500 & 5.47\end{array}$


U.S. DEPT. OF COMM.

BIBLIOGRAPHIC DATA

SHEET (See instructions)
1. PUBLICATION OR REPORT NO.

NBSIR 84-3015
2. Performing Organ. Report No.

3. Publication Date

August 1984

4. TITLE AND SUBTITLE

A Preliminary Investigation Into Using the Sun as a Source for G/T Measurements

5. AUTHOR(S)

William C. Daywitt

6. PERFORMING ORGANIZATION (If joint or other than NBS, see instructions)

7. Contract/Grant No.

NATIONAL BUREAU OF STANDARDS

DEPARTMENT OF COMMERCE

8. Type of Report \& Period Covered

WASHINGTON, D.C. 20234

9. SPONSORING ORGANIZATION NAIIE AND COMIFLETE ADCRESS (StreEt, CitY. StGTE, ZIP)

Same as 6

10. SUPPLEMENTARY NOTES

[] Document describes a computer program; SF-185, FIPS Software Summary, is attached.

11. ABSTRACT (A 200-word or less factual summary of most significant information. If document includes a significant bibliography or literature survey, mention it here)

This report describes a preliminary investigation into determining the solar flux density, the atmospheric correction factor, and the star shape correction factor for use in G/T measurements above $5 \mathrm{GHz}$. An estimate of errors is also included. Preliminary results show: an improved algorithm for determining diffusive and refractive attenuation; a viable technique for estimating the solar flux density from daily AFGL flux density measurements and a centimeter/millimeter wave spectrum function; and the possibility of reducing star shape correction factor errors by use of an effective solar $r f$ diameter.

12. KEY WORDS (Six to twelve entries; alphabetical order; capitalize only proper names; and separate key words by semicolons) atmospheric correction factor; earth terminal measurement system; error analysis; G/T; solar flux density; star shape correction factor

13. AVAILABILITY

X Unlimited

$\square$ For Official Distribution. Do Not Release to NTIS

$\square$ Order From Superintendent of Documents, U.S. Government Printing Office, Washington, D.C. 20402.

[X] Order From National Technical Information Service (NTIS), Springfield, VA. 2216I
14. NO. OF PRINTED PAGES

\section{4}

15. Price 


